\title{
Statistical Analysis of Tensor Fields
}

\author{
Yuchen Xie, Baba C. Vemuri, and Jeffrey Ho \\ Department of Computer and Information Sciences and Engineering \\ University of Florida
}

\begin{abstract}
In this paper, we propose a Riemannian framework for statistical analysis of tensor fields. Existing approaches to this problem have been mainly voxelbased that overlook the correlation between tensors at different voxels. In our approach, the tensor fields are considered as points in a high-dimensional Riemannian product space and accordingly, we extend Principal Geodesic Analysis (PGA) to the product space. This provides us with a principled method for linearizing the problem, and coupled with the usual log-exp maps that relate points on manifold to tangent vectors, the global correlation of the tensor field can be captured using Principal Component Analysis in a tangent space. Using the proposed method, the modes of variation of tensor fields can be efficiently determined, and dimension reduction of the data is also easily implemented. Experimental results on characterizing the variation of a large set of tensor fields are presented in the paper, and results on classifying tensor fields using the proposed method are also reported. These preliminary experimental results demonstrate the advantages of our method over the voxel-based approach.
\end{abstract}

\section{Introduction}

Tensor field data sets are quite commonly encountered in diffusion tensor imaging (DTI) [1] and Tensor-Based Morphormetry (TBM) [2]. Most tensor fields that have been reported in recent medical image analysis literature are fields of symmetric positive-definite matrices (SPDs), and this paper proposes a framework for statistical analysis on the space of these tensor fields.

The Riemannian geometry of the SPD matrices and its applications to medical image analysis problems that require statistical analysis of ensembles of SPD matrices has been the focus of intensive study in the past several years, e.g. [3 59]. In [5], Pennec et al. developed a Riemannian framework for computing statistics on SPD tensors. In [9], Schwartzman discussed the geometry of positive-definite matrices and studied probability distributions defined on SPD matrices. Principal Geodesic Analysis (PGA), as a generalization of Principal Component Analysis (PCA) for data on a Riemannian manifold, was introduced in [4]. In [3], Fletcher and Joshi described PGA-based methods for statistical analysis of diffusion tensors, in the context of computing (Karcher) mean of a collection of SPD matrices and characterizing their variance.

We remark that all these earlier works have focused on the statistical analysis of symmetric positive-definite matrices, and to the best of our knowledge there is no literature on statistical analysis of tensor fields where each field is treated in its entirety as a single entity. Although the methods for SPD matrices can be applied to study the 


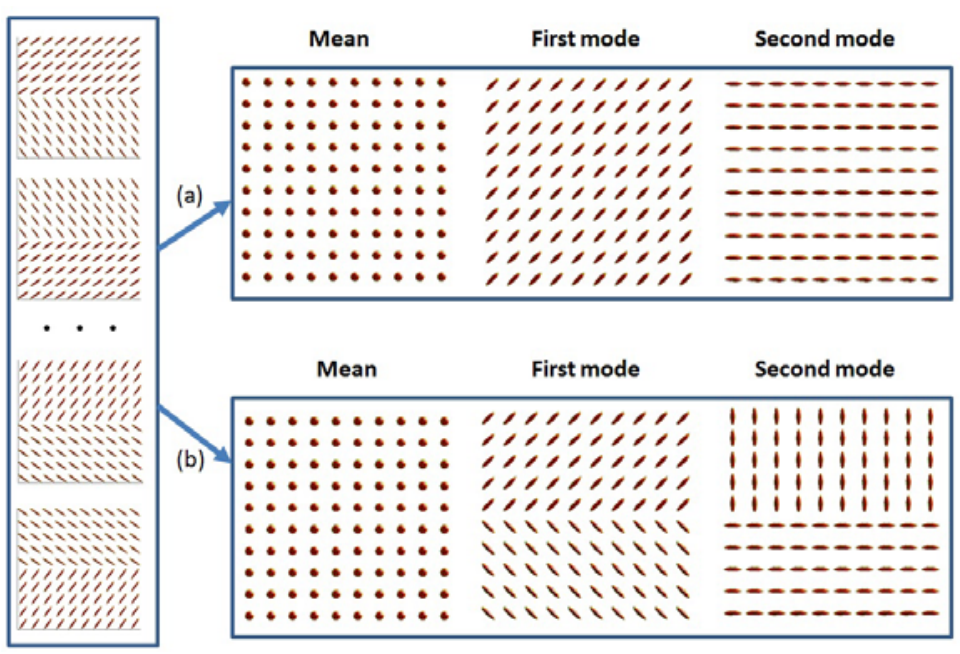

Fig. 1. Left column: Eight input tensor fields. (a) Mean, first mode and second mode of tensor fields computed using a pixel-based approach: means and modes are determined at each pixel independently using eight tensors. Note that the two modes are constant fields. (b) Mean, first mode and second mode of tensor fields computed using the proposed method.

statistics of tensor fields using a voxel-based approach where tensor fields are aligned and the statistics are gathered independently for every voxel, it is clearly insufficient and inadequate as it fails to capture global interaction patterns in the tensor fields. This point can be best illustrated using a simple example shown in Figure 1. Here, we generate four pairs of $10 \times 10$ tensor fields. Each tensor field contains two constant subfields occupying the top and bottom regions of the domain. Tensor fields in each pair differ by a reflection that swaps the top and bottom regions. For this collection of eight tensor fields, pixel-wise statistics fails to capture the global patterns in the tensor fields as the first and second modes are all constant fields. This result is not surprising as a pixelbased approach only considers tensors at each location independently, and it completely ignores the possible correlation between tensors at different locations.

In this paper, we propose a Riemannian framework for statistical analysis of a set of tensor fields that is capable of capturing the global correlation within the fields. Specifically, each tensor field can be represented by a point in the Riemannian product space. We extend Principal Geodesics Analysis (PGA) to Riemannian symmetric products, and this provides a principled method for linearizing the problem by mapping data (tensor fields) to a Euclidean space that is the tangent space at one specific point. The global correlations of the tensor field are then captured using Principle Component Analysis (PCA) in the tangent space, and the modes of variation for the tensor fields can be determined first in the tangent space followed by the exponential map. In addition, dimensionality reduction of data can also be efficiently implemented using PCA in the Euclidean space. This is particularly important as one major difficulty of working with the space of tensor fields is its dimension. For example, the space of $100 \times 100 \times 100$ 
tensor-valued images has $6 \times 10^{6}$ dimension, and dimensionality reduction is necessary for most applications and analysis. The proposed method is evaluated using OASIS dataset. We characterized the variation within a set of deformation tensor fields and applied our method to the tensor field classification problem. Preliminary experimental results have demonstrated the superiority of our method compared to the voxel-based approach.

\section{Statistical Analysis in the Space of Tensor Fields}

In this section, we present the details of statistical analysis in the space of tensor fields, and we will consider only fields of symmetric positive-definite matrices. Let $P(n)$ denote the space of $n \times n$ symmetric positive-definite matrices and $\operatorname{Sym}(n)$ denote the space of $n \times n$ symmetric matrices. A tensor field defined on a domain $\Omega$ in $\mathbb{R}^{K}$ is treated as a function $f: \Omega \rightarrow P(n)$. Since both diffusion tensor fields and deformation tensor fields used in medical imaging are almost always defined over a grid (pixels or voxels), $\Omega$ will be a collection of $m$ points in $\mathbb{R}^{K}$, and we identify the space of tensor fields on $\Omega$ with the product $P(n)^{m}=\underbrace{P(n) \times P(n) \times \cdots \times P(n)}_{m}$. Thus a tensor

field $\mathbf{X}$ in $P(n)^{m}$ is represented as an m-tuple $\left(X_{1}, X_{2}, \ldots, X_{m}\right)$, where each $X_{i}$ is a symmetric positive-definite matrix, the value of $\mathbf{X}$ at one point in $\Omega$.

\subsection{Geometry of Tensor Fields}

The space $P(n)$ is a symmetric Riemannian manifold [3] with $G L(n)$ as the symmetry group. This can be generalized directly to product spaces $P(n)^{m}$ using product Riemannian structure, and in particular, the Riemannian geodesic distances, log and exponential maps have closed-form expressions. Specifically, the group $G L(n)^{m}$ acts transitively on $P(n)^{m}$ with the action given by $\phi_{\mathbf{G}}(\mathbf{X})=\left(G_{1} X_{1} G_{1}^{T}, \ldots, G_{m} X_{m} G_{m}^{T}\right)$, where each $G_{i} \in G L(n)$ is a $n \times n$ invertible matrix and $X_{i}$ is a $n \times n$ positive-definite matrix. The tangent space of $P(n)^{m}$ at any point can be identified with $\operatorname{Sym}(n)^{m}$ since the tangent space of a product manifold is the product of tangent spaces. Let $\mathbf{Y}, \mathbf{Z} \in T_{\mathbf{M}} P(n)^{m}$ be two tangent vectors at $\mathbf{M} \in P(n)^{m}$. The product Riemannian metric gives the inner product between the two vectors as

$$
\langle\mathbf{Y}, \mathbf{Z}\rangle_{\mathbf{M}}=\sum_{i=1}^{m} \operatorname{tr}\left(Y_{i} M_{i}^{-1} Z_{i} M_{i}^{-1}\right) .
$$

Using this metric, the Riemannian exponential map at $\mathbf{M}$ maps the tangent vector $\mathbf{Y}$ to a point in $P(n)^{m}$

$$
\operatorname{Exp}_{\mathbf{M}}(\mathbf{Y})=\left(G_{1} \exp \left(G_{1}^{-1} Y_{1} G_{1}^{-T}\right) G_{1}^{T}, \ldots, G_{m} \exp \left(G_{m}^{-1} Y_{m} G_{m}^{-T}\right) G_{m}^{T}\right)
$$

where $G_{i} \in G L(n)$ such that $\mathbf{M}=\left(G_{1} G_{1}^{T}, \ldots, G_{m} G_{m}^{T}\right)$. Given $\mathbf{X} \in P(n)^{m}$, the log map at $\mathbf{M}$ is

$$
\log _{\mathbf{M}}(\mathbf{X})=\left(G_{1} \log \left(G_{1}^{-1} X_{1} G_{1}^{-T}\right) G_{1}^{T}, \ldots, G_{m} \log \left(G_{m}^{-1} X_{m} G_{m}^{-T}\right) G_{m}^{T}\right) .
$$

Using this definition of log map in $P(n)^{m}$, the geodesic distance between two tensor fields $\mathbf{M}$ and $\mathbf{X}$ is computed by 


$$
d(\mathbf{M}, \mathbf{X})=\left\|\log _{\mathbf{M}}(\mathbf{X})\right\|=\sqrt{\sum_{i=1}^{m} \operatorname{tr}\left(\log ^{2}\left(G_{i}^{-1} X_{i} G_{i}^{-T}\right)\right)} .
$$

\subsection{Statistics on the Space of Tensor Fields}

Using the formula above for the geodesic distance, we define the (intrinsic) mean of $N$ tensor fields as the tensor field that minimizes the sum of squared geodesic distances:

$$
\mathbf{M}=\arg \min _{M \in P(n)^{m}} \frac{1}{N} \sum_{i=1}^{N} d\left(M, \mathbf{X}_{i}\right)^{2} .
$$

The sum of squares on the RHS above can be re-written as a sum over all points in $\Omega$. This implies that the value of $\mathbf{M}(p)$ of $\mathbf{M}$ at one point $p \in \Omega$ is the usual Karcher mean in $P(n)$ of $\mathbf{X}_{1}(p), \cdots, \mathbf{X}_{N}(p)$. In particular, since the Karcher mean is unique on $P(n)$ [3], this shows that $\mathbf{M}$ will be unique as well, and it can be computed using an iterative algorithm similar to the one in [3].

After obtaining the intrinsic mean $\mathbf{M}$ of the input tensor fields $\mathbf{X}_{1}, \ldots, \mathbf{X}_{N}$, we will determine the modes of variation using PGA. Specifically, we use the log map to map all the tensor fields to the tangent space at $\mathbf{M}, x_{i}=\log _{\mathbf{M}}\left(X_{i}\right)$. This is a Euclidean space in which we can analyze the data points $x_{1}, \cdots, x_{N}$ using principal component analysis. We define the principal vectors $\mathbf{V}_{1}, \cdots, \mathbf{V}_{k}$ in $T_{\mathbf{M}} P(n)^{m}$ according to the following equations:

$$
\begin{array}{r}
\mathbf{V}_{1}=\arg \max _{\|\mathbf{V}\|=1} \sum_{i=1}^{N}\left\langle\mathbf{V}, \log _{\mathbf{M}}\left(\mathbf{X}_{i}\right)\right\rangle_{\mathbf{M}}^{2} \\
\mathbf{V}_{k}=\arg \max _{\|\mathbf{V}\|=1} \sum_{i=1}^{N} \sum_{j=1}^{k-1}\left\langle\mathbf{V}_{j}, \log _{\mathbf{M}}\left(\mathbf{X}_{i}\right)\right\rangle_{\mathbf{M}}^{2}+\left\langle\mathbf{V}, \log _{\mathbf{M}}\left(\mathbf{X}_{i}\right)\right\rangle_{\mathbf{M}}^{2}
\end{array}
$$

The orthonormal vectors $\mathbf{V}_{i}$ spanned a $K$-dimensional subspace $S_{K}$ that best approximates $x_{1}, \cdots, x_{N}$ in the least-squares sense, and they can be computed using PCA. By exponentiating vectors in $\mathbf{S}_{K}$, $\operatorname{Exp}_{\mathbf{M}}\left(\sum_{k=1}^{d} \alpha_{k} \mathbf{V}_{k}\right)$, where $\alpha_{k}$ tells the variation of $\mathrm{k} t h$ mode, we obtain the geodesic submanifold $\mathbf{S}_{K} \subset P(n)^{m}$ that can serve as a good approximation of the input tensor fields.

There are two important details that differ from the usual application of PGA [3]. First, except at the identity, the inner product defined in Equation 1 does not correspond to the standard Euclidean inner product, which is required for the familiar PCA algorithm. Therefore, we first transform the data to the tangent space at the identity, which is accomplished via the following transform, $\mathbf{X} \in T_{\mathbf{M}} P(n)^{m}$

$$
\phi_{\mathbf{G}^{-1}}(\mathbf{X}):\left(X_{1}, \cdots X_{m}\right) \longrightarrow\left(G_{1}^{-1} X_{1} G_{1}^{-T}, \ldots, G_{m}^{-1} X_{m} G_{m}^{-T}\right),
$$

where $\mathbf{G}=\left(G_{1}, \ldots, G_{m}\right)$ is such that $\mathbf{M}=\left(G_{1} G_{1}^{T}, \ldots, G_{m} G_{m}^{T}\right)$. Once the data have been mapped to $T_{\mathbf{I}} P(n)^{m}$, we can apply the usual PCA algorithm to obtain principal 
vectors $\mathbf{U}_{i}, i=1, \cdots, K$. They are then transformed back to $T_{\mathbf{M}} P(n)^{m}$ using $\mathbf{V}_{i}=$ $\phi_{\mathbf{G}}\left(\mathbf{U}_{i}\right)$. Due to the high-dimensionality of $P(n)^{m}$, we use the Gram matrix instead of the usual covariance matrix when computing the principal vectors in the tangent space, which is the approach used in many computer vision applications such as the Eigenfaces [8]. The complete algorithm is summarized in Algorithm One.

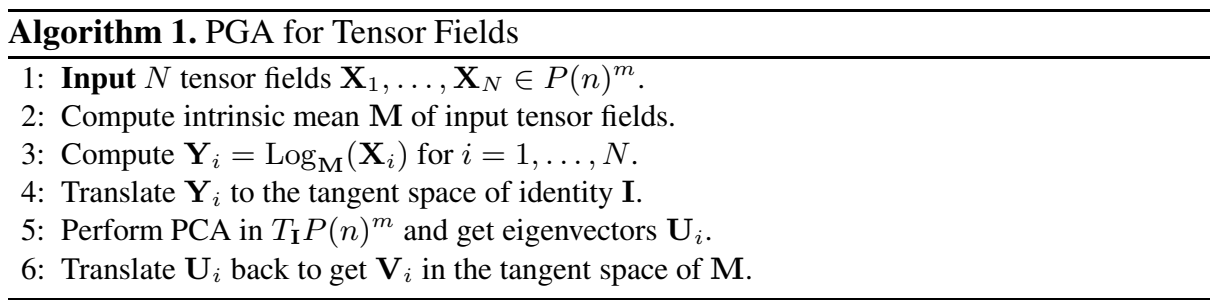

\subsection{Tensor Fields Classification}

We can formulate a tensor field classification algorithm using the principal directions and geodesic submanifolds. One common method for solving classification problems on Riemannian manifold is to map input data to the tangent space and do the classification in the tangent space [6]. However, this approach does not respect the geometry of the manifold as the geodesic distance between two points on the manifold are usually not the same or even commensurate with the distance between their images in the tangent space. A more principled approach is to use the distances to geodesic submanifolds as the feature for classification.

Assume a binary classification problem, and the training tensor fields are labelled as one of the two classes. For each label $k(k=1,2)$, we compute a low-dimensional geodesic submanifold $\mathbf{S}_{k}$ using training tensor fields with label $k$. For a test tensor field $\mathbf{X}$, we can determine its class by comparing the geodesic distances $d_{k}=$ $\min _{\mathbf{Y} \in \mathbf{S}_{k}} d(\mathbf{X}, \mathbf{Y})$. A tensor field is classified as belonging to class $k$ if $d_{k}$ is smaller than the other geodesic distance. The key step in this algorithm is to find the minimizer in $\mathbf{S}_{k}$ that gives the geodesic distance $d_{k}$. Since any point in the geodesic submanifold $\mathbf{S}_{k}$ can be written as $\operatorname{Exp}_{\mathbf{M}}\left(\sum_{i=1}^{d} \alpha_{i} \mathbf{V}_{i}\right)$, where $\mathbf{M}$ is the mean and $\alpha_{1}, \ldots, \alpha_{d}$ are real coefficients, $d_{k}$ can be solved via the following optimization problem in $\mathbb{R}^{d}$,

$$
\min _{\alpha_{1}, \ldots, \alpha_{d}} d\left(\mathbf{X}, \operatorname{Exp}_{\mathbf{M}}\left(\sum_{i=1}^{d} \alpha_{i} \mathbf{V}_{i}\right)\right)^{2} .
$$

Unfortunately, minimizing Equation 7 can be time-consuming for large tensor fields. Therefore, we approximate $d_{k}$ by the geodesic distance $d(\mathbf{X}, \mathbf{Z})$ between $\mathbf{X}$ and $\mathbf{Z}$ defined by

$$
\mathbf{Z}=\operatorname{Exp}_{\mathbf{M}}\left(\sum_{i=1}^{d} \mathbf{V}_{i}\left\langle\mathbf{V}_{i}, \log _{\mathbf{M}}(\mathbf{X})\right\rangle_{\mathbf{M}}\right) .
$$

That is, we obtain $\mathbf{Z}$ by first map $\mathbf{X}$ to the tangent space at $\mathbf{M}$ using Log map and project it onto the principal subspace $S_{d}$. The projection is then mapped down to the 
manifold using the exponential map to get $Z$. In our experiments discussed in the next section, the differences between the approximated distance and the one computed by the optimization are less than $1 \%$ and have no major influence on the classification results. We summarize the tensor fields classification algorithm in Algorithm Two.

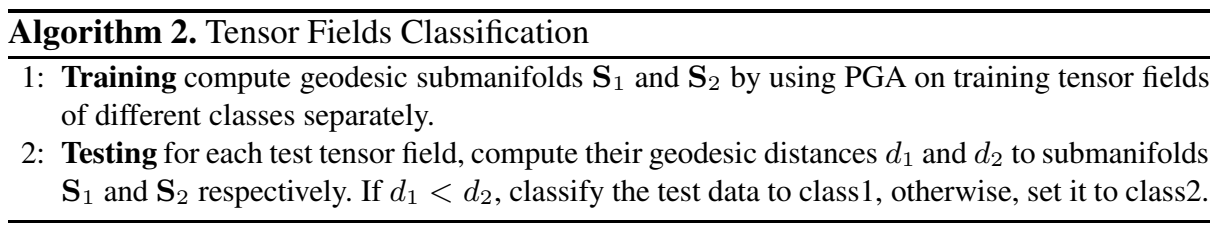

(a)
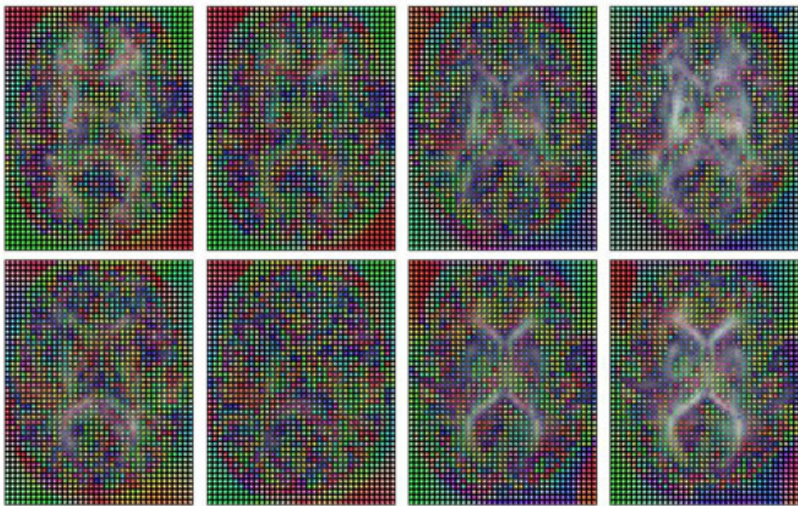
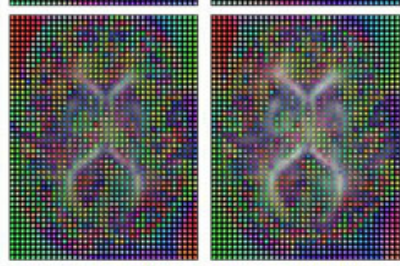

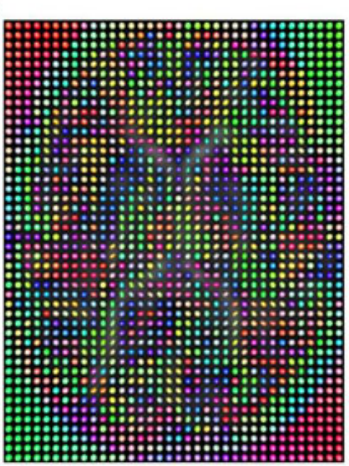

mean

Fig. 2. Statistical Analysis for deformation tensor fields from old age group. For better visualization, we downsample the images, only axial view is shown and set FA as the background in the display. (a) Tensor field variation along the first principal direction. From left to right, the coefficient $\alpha_{1}$ is $-2 \sigma_{1},-\sigma_{1}, \sigma_{1}, 2 \sigma_{1}$. (b) Tensor field variation along the second principal direction. From left to right, the coefficient $\alpha_{2}$ is $-2 \sigma_{2},-\sigma_{2}, \sigma_{2}, 2 \sigma_{2}$. Right column: Mean tensor field.

\section{Experimental Results}

The data used in our experiments are from the freely available Open Access Series of Imaging Studies (OASIS) MRI data set, which contains a cross-sectional collection of 416 subjects aged between 18-96 [7]. Each brain image has a resolution of $176 \times 208 \times$ 176 voxels. We divided 416 subjects three groups: young subjects (40 or younger), middle-aged subjects (between 40 and 60 years of age) and old subjects (60 or older). There is a subset of old subjects that were diagnosed with probable Alzheimer's Disease (AD). We compute the atlas for all the MR images in the OASIS data set using a groupwise nonrigid registration [10], and this also gives the the deformation field from each image to the atlas. For each voxel, we compute the Jacobian matrix $J$ of the deformation field and build the deformation strain tensor $S=\left(J^{T} J\right)^{1 / 2}$. This gives a strain tensor field for every subject. 


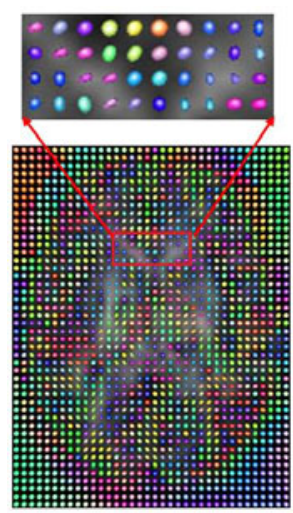

(a)

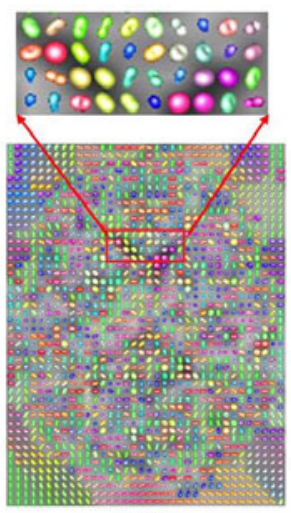

(b)

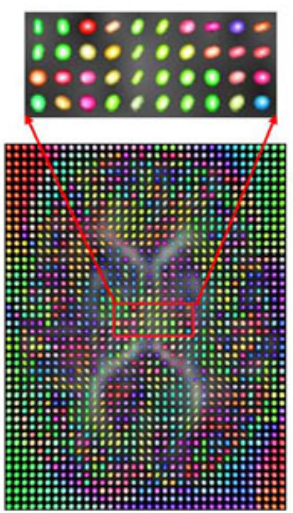

(c)

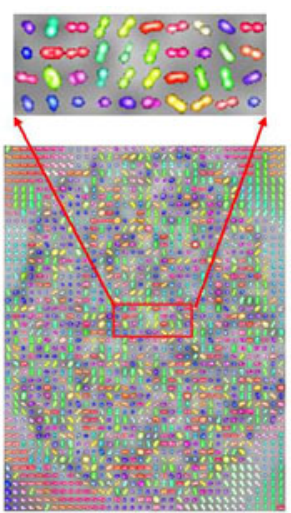

(d)

Fig. 3. Comparisons with voxel-based method. (a) and (c) are the first mode $\left(\sigma_{1}\right)$ and second mode $\left(\sigma_{2}\right)$ computed using Algorithm One. (b) and (d) are the first mode and the second mode computed using voxel-wise PGA. FA is used as the background in the display.

Table 1. Tensor Fields Classification on OASIS

\begin{tabular}{|c|c|c|c|c|}
\hline & Old vs. Young & Old vs. Middle & Middle vs. Young & AD vs. Control \\
\hline Nearest Neighbor & $92.43 \%$ & $87.74 \%$ & $78.42 \%$ & $84.29 \%$ \\
Submanifold Projection & $96.43 \%$ & $90.23 \%$ & $84.32 \%$ & $88.57 \%$ \\
\hline
\end{tabular}

In the first experiment, we characterize the variation in the tensor fields within an age group by computing the modes of variation using Algorithm-1. The dimension of the geodesic submanifold is set at 20 after examining the eigenvalue distribution. Figure 2 displays the mean tensor fields and the variations along the first two principal directions for the old group. The comparison with the mean and modes computed using the voxelbased approach in shown in Figure 3. We can clearly see that the modes computed using voxel-based method are fragmentary and they do not reflect the global structures of the tensor fields. This is not surprising because voxel-based method does not consider correlations between different voxels.

For the second experiment, we test our tensor-field classification algorithm. We randomly divide the brain images for each age group into four subsets. Images from one of the subsets are the test images, while other three subsets give the training images. The training images are used to compute the geodesic submanifolds $\mathbf{S}_{d}$, and we classify the test images for every pair of age groups using Algorithm 2. We use a four-fold cross-validation in the experiments to fully evaluate the algorithm on OASIS data set. We compared the performance of our algorithm with the nearest neighbor method that maps each tensor field to the tangent space of the mean. Low-dimensional feature vectors are generated using PCA projection, and the classification is done using nearest neighbor of these feature vectors. We have also tested the proposed algorithm on classification for healthy and diseased (Alzheimer) brain images. All results are tabulated in Table 1 The experimental results indicate that the deformation strain tensor fields 
do capture the subtle structural changes in the brain images across different age groups (and also for diseased samples), and the good classification results show the importance and value of doing statistical analysis on the space of tensor fields.

\section{Conclusions}

In this paper, we have presented the geometry of the space of tensor fields and proposed a framework for statistical analysis of a set of tensor fields. We have extended the PGA framework to the space of tensor fields considered as a Riemannian product space, and the modes of variation computed by the proposed algorithm capture the correlation between tensors at different locations. We have also proposed a novel tensor field classification algorithm using distances to the geodesic submanifolds as the main features for classification. Experimental results have shown that our approach provides a better characterization of the variation within a collection of tensor fields when compared to the voxel-based approach. In addition, good classification results on a large population of brain images have further validated the proposed framework.

\section{Acknowledgement}

This research is in part supported by the NIH grant EB007082 to BCV and the NSF grant IIS 0916001 to JH and BCV.

\section{References}

1. Basser, P.J., Mattiello, J., Le Bihan, D.: MR diffusion tensor spectroscopy and imaging. Biophysics Journal 66, 295-267 (1994)

2. Lepore, N., Brun, C., Chou, Y., Chiang, M., Dutton, R.A., Hayashi, K.M., Luders, E., Lopez, O.L., Aizenstein, H.J., Toga, A.W., Becker, J.T., Thompson, P.M.: Generalized Tensor-Based Morphometry of HIV/AIDS Using Multicariate Statistics on Deformation Tensors. IEEE Transations on Medical Imaging (2008)

3. Fletcher, P.T., Joshi, S.: Riemannian Geometry for the Statistical Analysis of Diffusion Tensor Data. Signal Processing (2007)

4. Fletcher, P.T., Lu, C., Pizer, S.M., Joshi, S.: Principal Geodesic Analysis for the Study of Nonlinear Statistics of Shape. IEEE Transactions on Medical Imaging (2004)

5. Pennec, X., Fillard, P., Ayache, N.: A Riemannian Framework for Tensor Computing. International Journal of Computer Vision (2006)

6. Wu, J., Smith, W.A.P., Hancock, E.R.: Gender Classification using Shape from Shading. British Machine Vision Conference (2007)

7. Marcus, D.S., Wang, T.H., Parker, J., Csernansky, J.G., Moris, J.C., Buckner, R.L.: Open Access Series of Imaging Studies (OASIS): Cross-Sectional MRI Data in Young, Middle Aged, Nondemented, and Demented Older Adults. Journal of Cognitive Neuroscience (2007)

8. Turk, M.A., Pentland, A.P.: Face Recognition Using Eigenfaces. In: IEEE Conference on Computer Vision and Pattern Recognition (1991)

9. Schwartzman, A.: Random Ellipsoids and False Discovery Rates: Statistics for Diffusion Tensor Imaging Data. Ph.D. dissertation, Dept. of Statistics. Stanford University (2006)

10. Joshi, S., Davis, B., Jomier, M., Gerig, G.: Unbiased Diffeomorphic Atlas Construction for Computational Anatomy. NeuroImage (2004) 